\title{
The striated muscles in pulmonary arterial hypertension: adaptations beyond the right ventricle
}

\author{
Emmy Manders ${ }^{1,2}$, Silvia Rain ${ }^{1}$, Harm-Jan Bogaard ${ }^{1}$, M. Louis Handoko ${ }^{1,3}$, \\ Ger J.M. Stienen ${ }^{2,4}$, Anton Vonk-Noordegraaf ${ }^{1}$, Coen A.C. Ottenheijm ${ }^{2}$ and \\ Frances S. de $\operatorname{Man}^{1}$
}

\author{
Affiliations: \\ ${ }^{1}$ Dept of Pulmonology, VU University Medical Center, Institute for Cardiovascular Research, Amsterdam, The \\ Netherlands. \\ ${ }^{2}$ Dept of Physiology, VU University Medical Center, Institute for Cardiovascular Research, Amsterdam, The \\ Netherlands. \\ ${ }^{3}$ Dept of Cardiology, VU University Medical Center, Institute for Cardiovascular Research, Amsterdam, The \\ Netherlands.
}

${ }^{4}$ Dept of Physics and Astronomy, VU University, Amsterdam, The Netherlands.

\section{Correspondence:}

Frances S. de Man, Dept of Pulmonary Diseases, Institute for Cardiovascular Research, VU University Medical Center, de Boelelaan 1017, 1081 HV Amsterdam, The Netherlands.

E-mail: fs.demandvumc.nl.

ABSTRACT Pulmonary arterial hypertension (PAH) is a fatal lung disease characterised by progressive remodelling of the small pulmonary vessels. The daily-life activities of patients with PAH are severely limited by exertional fatigue and dyspnoea. Typically, these symptoms have been explained by right heart failure. However, an increasing number of studies reveal that the impact of the PAH reaches further than the pulmonary circulation. Striated muscles other than the right ventricle are affected in $\mathrm{PAH}$, such as the left ventricle, the diaphragm and peripheral skeletal muscles. Alterations in these striated muscles are associated with exercise intolerance and reduced quality of life. In this Back to Basics article on striated muscle function in $\mathrm{PAH}$, we provide insight into the pathophysiological mechanisms causing muscle dysfunction in PAH and discuss potential new therapeutic strategies to restore muscle dysfunction.

@ERSpublications

RV, LV, diaphragm and peripheral muscle dysfunction contributes to reduced quality of life in PAH patients http://ow.ly/NAyZP 


\section{Introduction}

Pulmonary arterial hypertension (PAH) is a fatal lung disease with a poor prognosis. Although the origin of the disease is progressive remodelling of the small pulmonary vessels, patients eventually die of right heart failure. The right ventricle (RV), which pumps blood into the lungs for oxygenation, has to face an enormous increase in pressure in PAH. Despite initial adaptations, eventually, the RV is not capable of sustaining the chronic pressure overload, and will dilate and fail [1-4].

An increasing number of studies reveal that the impact of $\mathrm{PAH}$ reaches further than the pulmonary circulation. Striated muscles other than the RV are affected in PAH, such as the left ventricle (LV), the diaphragm and peripheral skeletal muscles. Alterations in these muscle types are closely associated with clinical worsening, exercise intolerance and reduced quality of life [5-9]. Therefore, it is of utmost importance to obtain more insight into the pathophysiological mechanisms causing muscle dysfunction in PAH.

In this Back to Basics article on striated muscle function in $\mathrm{PAH}$, we provide: 1) background information on normal striated muscle structure and function; 2) an overview of cardiac muscle alterations in $\mathrm{PAH}$; 3) an overview of the changes in skeletal muscle function in $\mathrm{PAH}$; and 4) a discussion of potential new therapeutic strategies to restore or even prevent striated muscle dysfunction in $\mathrm{PAH}$.

\section{Striated muscle structure and function: a brief recap}

To better understand the contribution of striated muscle dysfunction in the setting of PAH, we will first briefly describe general striated muscle structure and function. The heart and skeletal muscles are built from muscle fibres (i.e. myocytes). Myocytes are composed of repeating structures called sarcomeres, which are the smallest contractile units in a muscle and give rise to the striated appearance of myocytes. Sarcomeres are composed of three filaments: the thin filament, the thick filament and the elastic filament (figure 1a). The thin filaments are composed of actin molecules with a troponin complex and a tropomyosin strand. The thick filaments are mainly composed of myosin molecules with myosin heads extending out to the sides, which can bind to the actin molecules of the thin filament when the muscle is activated. The elastic filament plays an important role in the maintenance of the structural integrity of the sarcomere and consists of the giant sarcomeric protein titin [11].

Muscle contraction occurs in response to an action potential in the plasma membrane. The electrical activity in the plasma membrane triggers the release of large quantities of calcium from the sarcoplasmic reticulum (SR) via the ryanodine receptors. Calcium binds to the troponin complex on the thin filaments. This leads to a conformational change of tropomyosin, thereby unblocking the actin binding sites. Exposure of these sites allows myosin heads to bind to actin and form a cross-bridge. The cross-bridge will move, thereby shortening the sarcomere and the muscle (figure 1a). At the end of contraction, calcium is pumped back into the SR by the sarco/endoplasmic reticulum calcium ATPase pump and the relaxation phase is initiated [10]. Relaxation of the sarcomeres is further determined by the elastic properties of titin. Compliant titin molecules result in proper relaxation of the muscles, whereas stiff titin molecules limit relaxation [11].

Sarcomere function can be modulated by post-translational or transcriptional modifications. Post-translational modifications, particularly phosphorylation, regulate cardiomyocyte contractile function and stiffness. Transcriptional changes influence, for example, myosin heavy chain isoform expression, the development of fibrosis and protein content [12].

Cardiac and skeletal muscles remodel in response to changes in demand or activity. For example, during sport, a chronic increase in cardiac function and/or skeletal muscle training leads to cardiac and skeletal muscle hypertrophy $[13,14]$, while reductions in cardiac load or muscle disuse lead to cardiac and skeletal muscle atrophy $[15,16]$. The remodelling is not limited to muscle size but also includes alterations in the contractility and passive stiffness of the sarcomere and muscle.

\section{Cardiac dysfunction in PAH is not limited to the right ventricle}

As a consequence of pulmonary vascular proliferation and remodelling, pulmonary vascular resistance increases in PAH patients. This results in an increased demand on the RV, leading to RV remodelling. This remodelling includes excessive interstitial fibrosis and cardiomyocyte hypertrophy; consequently, the RV develops both systolic and diastolic dysfunction. For further details on RV dysfunction in PAH, please see reviews $[4,17,18]$.

\section{Structural and contractile alterations in the LV}

$\mathrm{RV}$ remodelling in PAH patients also affects LV function. LV dysfunction in PAH patients is characterised by reduced ejection fraction and impaired diastolic function, and some studies report a reduction in LV free wall mass in PAH patients [6, 19-22]. However, the pathophysiology of LV dysfunction in PAH is 
a)
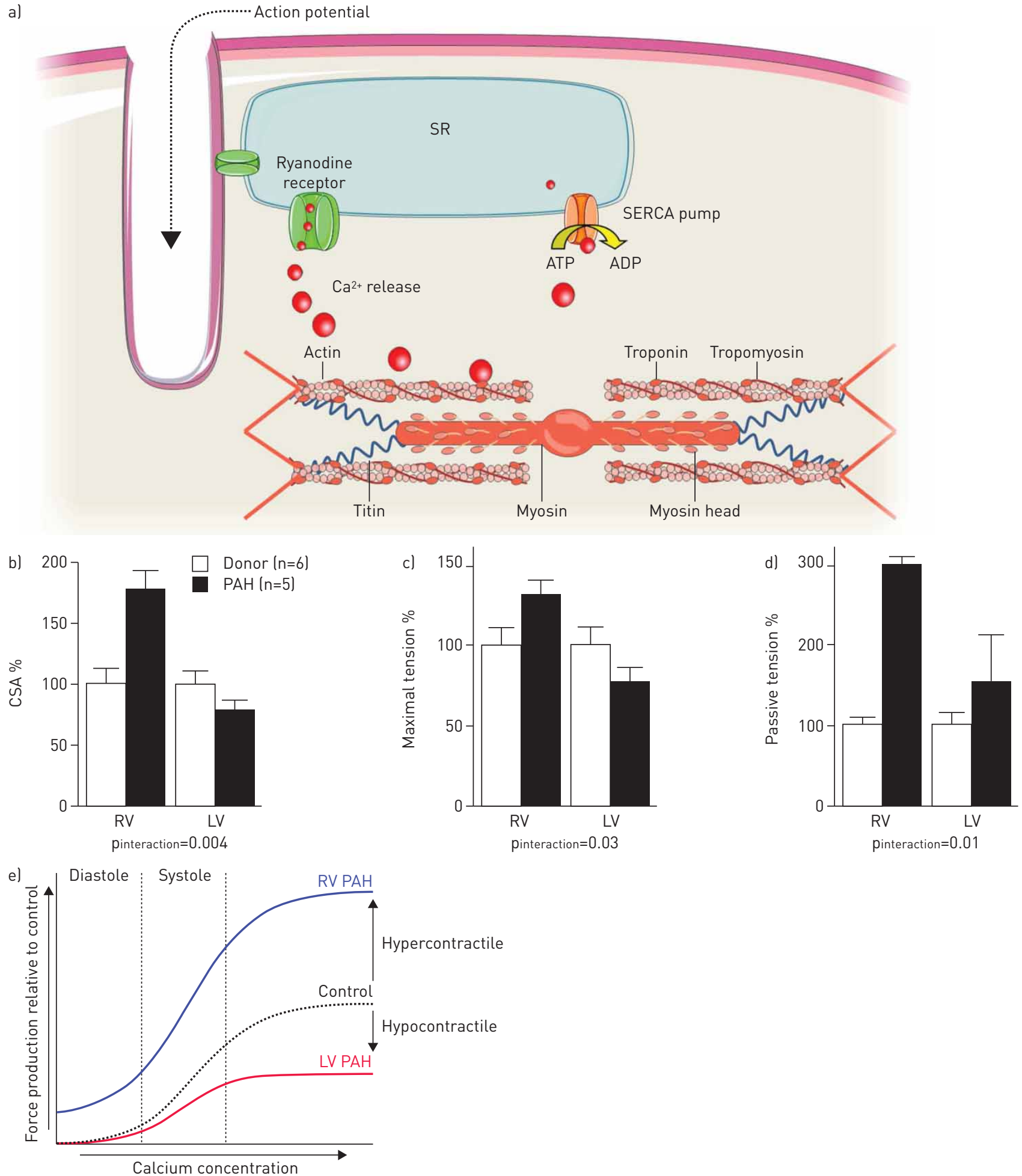

FIGURE 1 a) Muscle function. Muscle contraction starts when an action potential reaches the T-tubules. This leads to the release of large quantities of calcium from the sarcoplasmic reticulum (SR) via the ryanodine receptors. When calcium enters the sarcomeres, it binds to the troponin complex on the thin filaments. This leads to a conformation change of tropomyosin, thereby unblocking the actin-binding sites. Exposure of these sites allows myosin heads from the thick filament to bind to actin and form a cross-bridge. The cross-bridge will move, thereby shortening the muscle and producing tension in the thin filament. At the end of contraction, $\mathrm{Ca}^{2+}$ is pumped back into the SR by the sarco/endoplasmic reticulum calcium ATPase (SERCA) pump. Created using Servier Medical Art (www.servier.com). b-d) Comparison of the right ventricle (RV) and left ventricle (LV) of the same pulmonary arterial hypertension (PAH) patients. All parameters are expressed relative to donor values of the specific ventricle. b) Cardiomyocyte cross-sectional area (CSA) was increased in RV cardiomyocytes, while it was decreased in the LV of PAH patients. c) Maximal tension (i.e. force normalised to CSA) was increased in RV cardiomyocytes, while it was decreased in LV cardiomyocytes of PAH patients. d) Passive tension was greatly increased in the RV, while passive tension was unchanged in LV cardiomyocytes. Data from $[1,5]$. e) Relative force production of RV and LV cardiomyocytes of PAH patients compared with control. RV cardiomyocytes are hypercontractile, while LV cardiomyocytes are hypocontractile compared with control subjects. 
incompletely understood. Recently, we obtained unique LV biopsies from PAH patients, and performed detailed analyses of LV cardiomyocyte structure, function and protein composition [5]. Our data showed that PAH LV cardiomyocyte cross-sectional area (CSA) was $~ 30 \%$ smaller than control cardiomyocytes, indicating severe LV atrophy in PAH patients. In addition, LV contractile function was reduced, even after correction for differences in cardiomyocyte CSA. This study indicated that, in addition to atrophy, intrinsic dysfunction of the sarcomeres limits the force-generating capacity of PAH patients' LV cardiomyocytes [5].

Clinically, this may explain the occurrence of LV failure in PAH patients after lung transplantation. RV afterload is restored after a lung transplant, and the hypertrophic and hypercontractile RV vigorously pumps blood into the now low-resistance pulmonary circulation. The LV, which had adapted to a low filling state, may not be able to cope with the sudden increase in filling and might fail [23-26].

\section{Underlying aetiology of LV dysfunction}

Alterations in cardiac muscle function in PAH may be explained by systemic factors and local (loading) factors.

\section{Systemic factors}

To compensate for the low cardiac output, neurohormonal systems are upregulated in PAH patients [27, 28]. However, chronic neurohormonal stimulation leads to a compensatory down regulation of $\beta$-adrenergic receptors leading to disturbed protein kinase A-mediated phosphorylation of contractile proteins [2, 29]. Reduced contractile protein phosphorylation affects cardiomyocyte contractility and relaxation. Indeed, in both the RV and LV of PAH patients key contractile proteins are hypophosphorylated, resulting in an increase in calcium sensitivity $[1,2,5]$. Several studies have demonstrated increased expression of systemic inflammatory markers in PAH patients [30-32]. Inflammation may amplify the formation of myocardial fibrosis and might affect contractile function by altering intracellular calcium transport and signal transduction [33]. In addition, it could be speculated that this contributes to the higher mortality and lower cardiac output in patients with systemic sclerosis PAH $[34,35]$.

\section{Local factors}

We propose that in addition to systemic factors, changes in load contribute substantially to the observed RV and LV dysfunction in PAH. The ventricles are not separate entities: the functions of the two ventricles are inextricably linked. The RV and LV are intimately attached through common muscle fibres and the interventricular septum, and share the pericardial space. Thus, adaptation of the RV induces alterations in the demand placed on the LV. An increase in RV afterload, as occurs in PAH patients, leads to a decrease in RV output. The RV and LV are serially linked; consequently, LV filling is reduced [22, 36, 37]. In addition, direct ventricular interaction might further impair LV filling. Leftward septum bulging is frequently observed in PAH patients $[6,19,38]$. This is caused by prolonged RV shortening, pushing the septum towards the LV during early diastole, hampering LV filling [38]. As the contractility of cardiomyocytes is sensitive to changes in demand and load $[14,15]$, the reduced filling of the LV in PAH patients might affect the function of LV cardiomyocytes.

Exciting new data where we have compared RV and LV cardiomyocyte adaptation in the same PAH patients further emphasises the importance of loading on RV and LV function $[1,5]$. As shown in figure 1b, RV cardiomyocyte CSA increased almost two-fold, while LV cardiomyocyte CSA decreased. This was paralleled by opposing results in maximal force development, even after correction for CSA (figure 1c). Furthermore, passive tension, a reflection of diastolic stiffness, increased three-fold in the RV, whereas LV passive tension was unchanged (figure 1d). Therefore, RV cardiomyocytes are hypercontractile, whereas LV cardiomyocytes are hypocontractile, in PAH patients (figure 1e). As the load imposed on the ventricles is also in opposite direction, this strengthens our proposition that load is a major contributor of cardiomyocyte remodelling in PAH.

\section{Skeletal muscle dysfunction in PAH: triggered by systemic or local factors?}

At rest, more than $20 \%$ of the cardiac output (CO) goes to the skeletal muscles. This percentage can increase up to $84 \%$ upon extreme physical exertion [39]. Cardiac function is therefore a very important determinant of exercise capacity. As described above, both RV and LV function are hampered in PAH patients, which results in a reduced capacity for stroke volume to augment $\mathrm{CO}$ during exercise. In addition, PAH patients fail to maximally increase heart rate during exercise. This chronotropic impairment in PAH patients is related to downregulation of $\beta$-adrenergic activity and is a reflection of disease severity [40-42]. Furthermore, oxygen saturation decreases during exercise in PAH patients [43]. Together with the reduction in CO, oxygen supply to the skeletal muscles might be limited, which may result in muscle dysfunction. 
Structural and contractile alterations in the skeletal muscles

Leg fatigue and dyspnoea are the dominant symptoms causing $\mathrm{PAH}$ patients to stop exercise, implying skeletal muscle dysfunction [42]. Maximal volitional and nonvolitional strength of both the inspiratory and quadriceps muscles are reduced in PAH patients, a reduction that correlates with exercise capacity [7, $9,44,45]$. Maximal muscle strength does not depend directly on oxygen supply, indicating that the intrinsic capacity of the skeletal muscle to generate force is impaired. Forearm grip strength correlates with maximal inspiratory pressure, suggesting the presence of a generalised muscle weakness in PAH patients [46]. However, impaired oxygen supply to the muscles due to lowered $\mathrm{CO}$ could also contribute to muscle weakness and fatigue during exercise. To discriminate between intrinsic muscle weakness and limitations in oxygen supply/extraction of the muscles, further experiments are needed; for example, by measuring exercise capacity and muscle strength under normoxia and hyperoxia (100\% oxygen) in combination with measurements of oxygen transport and metabolism [47] of the inspiratory and peripheral muscles in PAH patients and animal models. Inspiratory muscle strength largely depends on the function of the diaphragm. Diaphragm muscle fibres in PAH are atrophied and hypocontractile [8, 48-50]. More importantly, these changes also translated to in vivo reductions of inspiratory muscle contractility [50].

As illustrated in table 1, peripheral muscle structural and contractile alterations are less clear. Inconsistent data with respect to muscle fibre CSA, fibre-type distribution and capillary density are found in both PAH patients as well as animal models of pulmonary hypertension (PH) [8, 9, 49, 51-54]. In addition, in $\mathrm{PH}$ rats, no change in force-generating capacity was found, while in PAH patients, maximal tension was reduced $[8,49,55]$. Differences between experimental and patient data may be explained by differences in disease duration, i.e. $\sim 4$ weeks for animal models versus years for patients. However, also within animal or patient studies, inconsistencies have been observed. Thus, the underlying cause of the reduction in peripheral muscle strength is still not completely clear but might involve atrophy, sarcomere dysfunction, a shift towards more fast-twitch muscle fibres and capillary rarefaction.

\section{Underlying aetiology of skeletal muscle dysfunction}

In patients with chronic obstructive pulmonary disease (COPD) and chronic heart failure (CHF), similar alterations in inspiratory and peripheral muscles have been found [56]. There are even indications that the diaphragm is more weakened in $\mathrm{PAH}$ patients than in CHF patients [7, 44]. These similarities suggest that a general underlying mechanism might be at play. We propose that systemic factors may affect the balance between protein synthesis and protein degradation, leading to a systemic myopathy, but that changes in muscle activity are an additional trigger to induce skeletal muscle dysfunction.

\section{Systemic factors}

Besides a diminished oxygen supply to the skeletal muscles as a result of cardiac dysfunction, other systemic factors can affect muscle function. Levels of pro-inflammatory cytokines, such as interleukin (IL)-1, IL-6 and tumour necrosis factor- $\alpha$, are elevated in the systemic circulation of PAH patients [30, 31, 57]. Inflammatory cytokines affect muscle function by upregulation of proteolysis via the ubiquitin proteasome system and also induces contractile dysfunction [58,59]. Both in the diaphragm and quadriceps muscle of PAH patients and $\mathrm{PH}$ rats, increased levels of atrogin-1 and muscle RING finger protein (MuRF)-1 were found, which are key markers of proteolytic activity in muscle $[8,60]$. This leads to a reduction in contractile protein content and muscle fibre atrophy. Neurohormonal overstimulation might also affect muscle function by inducing changes in muscle metabolism and muscle regenerating capacity $[61,62]$.

Local factors

Skeletal muscles are very sensitive to changes in activity and load, and remodel accordingly $[13,16,63]$. In $\mathrm{PAH}$ patients, the activity of inspiratory and peripheral muscles changes to opposite direction. PAH

TABLE 1 Inspiratory and peripheral muscle structural and contractile alterations

\begin{tabular}{lccccc} 
& \multicolumn{2}{c}{ Inspiratory muscle } & & \multicolumn{2}{c}{ Peripheral muscle } \\
\cline { 2 - 3 } \cline { 5 - 6 } & Animal & Human & & Animal & Human \\
\cline { 5 - 6 } Muscle fibre size & $\downarrow[8,48]$ & $\downarrow[8],=[50]$ & & $=[8,51], \downarrow[52]$ & $=[8,9,53], \downarrow[54]$ \\
Maximal tension & $\downarrow[8,48,49]$ & $\downarrow[8,50]$ & & $=[8,49]$ & \\
Fast-twitch muscle fibres \% & $=[8]$ & $=[50]$ & & $\uparrow[51],=[52]$ & $\uparrow[9,54],=[53]$ \\
Capillary density & $=[8]$ & & & $=[52]$ & $\downarrow[53],=[9]$ \\
\hline
\end{tabular}

$\downarrow$ : decrease; =: no change; $\uparrow$ : increase. 
patients hyperventilate during exercise, at rest and sometimes even during sleep, placing an increased demand on the inspiratory muscles [64]. However, peripheral muscle activity might decrease, as physical activity declines with disease progression.

In $\mathrm{PH}$ rats, muscle fibre CSA and contractility were unaltered in peripheral muscle while both were reduced in the diaphragm muscle $[8,49]$. Similarly, in two end-stage PAH patients, quadriceps muscle fibre CSA was unaltered, while diaphragm muscle fibre CSA was reduced by $\sim 75 \%$ [8]. In addition, maximal tension was lower in muscle fibres of the diaphragm of chronic thromboembolic $\mathrm{PH}$ patients compared with those of a noninspiratory muscle of the same patient, suggesting specific weakening of the diaphragm muscle [50].

The diaphragm might be more vulnerable to changes in activity than the peripheral muscles. The activation of the diaphragm is very unique in relation to other skeletal muscles. Its normal daily duty cycle (ratio of active to inactive times) is $\sim 45 \%$, while it is $\sim 15 \%$ in peripheral muscles [65]. The overstimulation of the diaphragm might lead to a greater and faster development of an imbalance between protein synthesis and protein degradation caused by systemic factors and, consequently, muscle dysfunction. Peripheral muscles might be less vulnerable to the systemic factors as muscle activity is lower. However, muscle disuse or unloading can by itself also induce contractile dysfunction and muscle fibre atrophy [16, 63]. A specific feature of skeletal muscle disuse and unloading is a shift towards more fast-twitch muscle fibres [16, 63]. This fibre-type shift has also been observed in some PAH patients, strengthening our proposition that muscle activity might contribute to peripheral muscle dysfunction in PAH. In addition, fibre-type shifting has also been observed in COPD and CHF patients, conditions which are also associated with reduced activity of peripheral muscles and increased activity of the diaphragm [56]. However, the inconsistent results on peripheral muscle function and structure in $\mathrm{PAH}$ might suggest that this depends more on individual variations in physical activity, disease duration and/or disease severity.

In conclusion, the differences between inspiratory and peripheral muscle adaptation suggest that the alterations are not only caused by systemic effects. Differences in muscle activity of the inspiratory and peripheral muscles are most likely to be an additional trigger amplifying skeletal muscle dysfunction in PAH.

\section{Enclosing novel treatment targets}

The combination of a reduction in cardiac output, reduced oxygen supply to the muscles and skeletal muscle weakness leads to exercise intolerance in PAH-patients. Current disease-targeted treatments are insufficient to prevent progression of right heart failure. In addition, vasodilators might improve oxygen supply to the muscles and, thereby, muscle function but they do not sufficiently restore exercise capacity. Therefore, treatment strategies aimed at improving exercise capacity have gained importance. An overview of specific muscle treatment targets is illustrated in figure 2 .

\section{Improving endurance capacity \\ Exercise training}

Although it was initially thought that exercise training (ExT) could be detrimental for PAH patients, it is now considered a valuable to improve exercise capacity and quality of life. For a complete overview of ExT

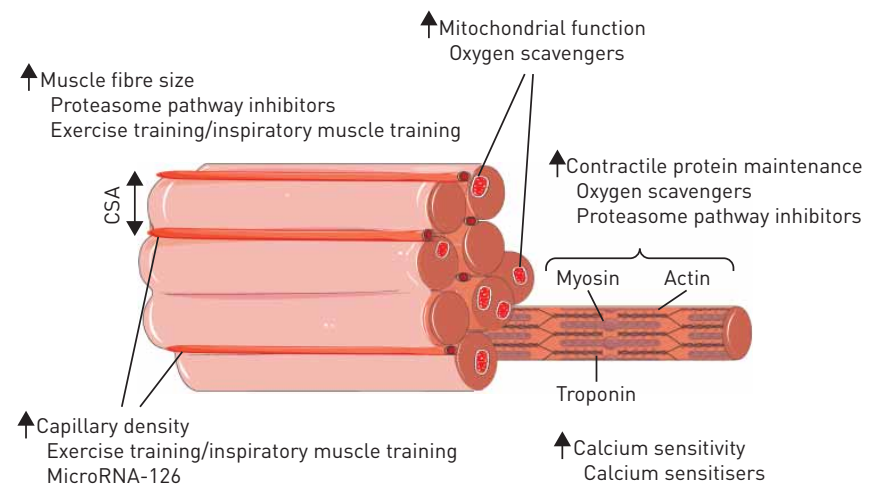

FIGURE 2 Schematic representation of muscle fibres, including capillaries, mitochondria and contractile proteins. Depicted are the points of application of new therapeutic options specifically targeting muscle function. Exercise and inspiratory muscle training may increase muscle fibre cross-sectional area (CSA) and capillary density. Proteasome inhibitors can improve muscle fibre CSA and contractile protein content. Oxygen scavengers can reduce oxidative stress, which might improve contractile protein and mitochondrial function. Calcium sensitisers could improve submaximal force-generating capacity and might improve muscle neuromechanical efficiency. Created using Servier Medical Art (www.servier.com). 
in PAH patients, see the review by ZAFrir et al. [66]. Large improvements in 6-min walk test (6MWT) distance were reported in several studies after only 3 weeks of ExT [67-71]. However, most of these programmes included walking training, which may directly act upon 6MWT distance. Nevertheless, improvements in peak maximal heart rate, peak oxygen uptake and maximal work load have also been observed after 12 weeks of training. De MAN et al. [72] demonstrated that exercise training also specifically alters quadriceps muscle function and morphology. Improvements in aerobic capacity by increased capillarisation and oxidative enzyme activity were found in the quadriceps muscle of PAH patients after training. These improvements were in line with the increase in quadriceps muscle endurance [72]. Others found a reduction in fast-twitch muscle fibres and a potential increase in capillary density in the quadriceps of PAH patients after ExT [73].

\section{Inspiratory muscle training}

With the inclusion of inspiratory muscle training (IMT) in the ExT programme, inspiratory muscle weakness can be targeted. Improvements in inspiratory muscle strength were reported in PAH patients but also in a randomised controlled trial of IMT alone in CHF patients $[69,74]$. In addition, no adverse effects have been reported in CHF patients, indicating that IMT is a safe tool to improve inspiratory muscle strength [75]. Furthermore, IMT and ExT reduce sympathetic drive, which might lead to improvements in cardiac function and respiratory drive [76]. Most importantly, $\mathrm{CHF}$ and $\mathrm{PAH}$ patients report a better quality of life and decreased sensation of dyspnoea on exertion after IMT [69, 70, 74].

Nevertheless, the optimal ExT intensity and strategy is not clear. Larger, long-term, multicentre studies are needed to evaluate the optimal protocol for exercise training. These protocols should include muscle function and morphological measurements to directly evaluate the effect of ExT on muscle function and exercise capacity.

\section{Improving muscle structure and metabolism}

Exercise training might not be feasible for all patients due to, for example, haemodynamic instability [77]. Therefore, other strategies to improve muscle function are necessary.

\section{Proteasome pathway inhibitors}

In $\mathrm{PAH}$, there is an imbalance between protein synthesis and degradation in peripheral and respiratory muscles, as demonstrated by increased expression of atrogin-1 and MuRF-1 [8,54]. In a mouse model of inflammation and immobilisation, it was shown that inhibitors of the proteasome pathway attenuate the expression of atrogin-1 and MuRF-1, leading to restoration of muscle fibre CSA [78]. Thus, treating PAH patients with proteasome pathway inhibitors might lead to improvements in muscle fibre size and contractile function $[79,80]$. Currently, bortezomib is in a phase I clinical trial for patients with dysferlinopathy to prevent muscle damage.

\section{MicroRNA-126}

For proper muscle function, the increase in muscle fibre size should be accompanied by an increase in capillarisation. Several studies have shown reduced capillary density in striated muscles of PAH patients $[53,77,81]$. РотUs et al. [53] recently demonstrated that capillary rarefaction in PAH patients was caused by downregulation of microRNA-126. Restoration of microRNA-126 in a rat model led to increased capillary density and improved endurance capacity. Therapeutic microRNA expression modulation is in clinical trials for various diseases. Thus, modulation of microRNA-126 expression might be a new, attractive therapeutic target to specifically improve capillary rarefaction in $\mathrm{PAH}$.

\section{Oxygen scavengers}

The reduction in oxygen supply and capillary rarefaction could lead to oxidative stress within the muscles. Oxidative stress induces protein breakdown and mitochondrial dysfunction or damage. Oxygen scavengers, such as $\mathrm{N}$-acetylcysteine, are able to reduce the levels of reactive oxygen species, thereby potentially reducing protein breakdown and ameliorating mitochondrial function [82]. $\mathrm{N}$-acetylcysteine is currently in several phase I and II clinical trials to prevent muscle oxidative stress in, for example, elderly, dialysis and cancer cachexia patients.

\section{Improving sarcomere function}

The aforementioned therapeutic options target muscle structure and metabolic pathways. However, sarcomere dysfunction is also an important contributor to weakness of the LV, diaphragm and quadriceps muscle in PAH patients, making the sarcomere an attractive target for treatment. 
FIGURE 3 Schematic overview of the muscular adaptations involved in pulmonary arterial hypertension (PAH) contributing to exercise intolerance. RV: right ventricle; LV: left ventricle.

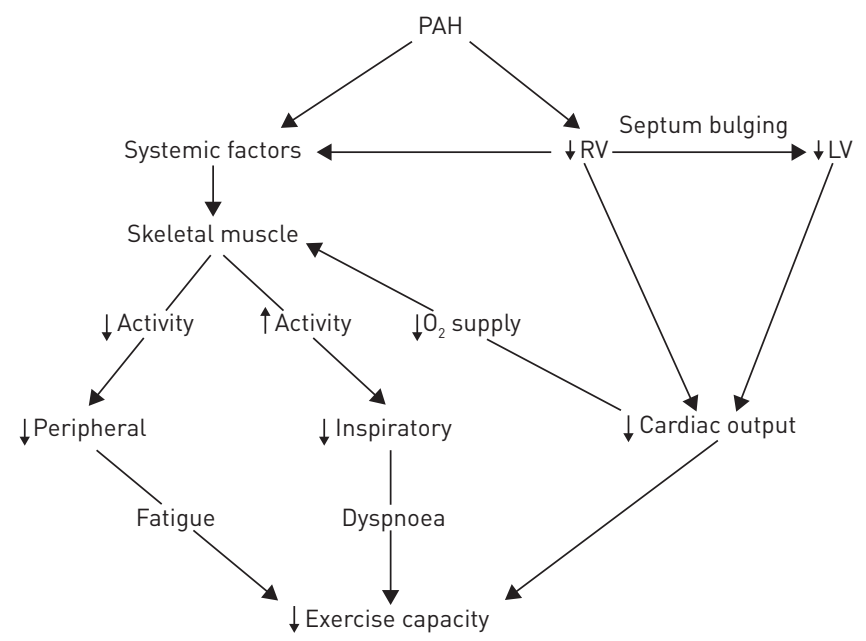

\section{Calcium sensitisers}

Calcium sensitisers are small-molecule drugs that slow the dissociation of calcium from the troponin complex, thereby stabilising the open conformation of the troponin/tropomyosin complex to enhance cross-bridge formation. Currently, levosimendan is the only clinically available calcium sensitiser. However, levosimendan targets slow-twitch muscle fibres and also affects cardiac muscle [83, 84]. As calcium sensitivity in the ventricles is already increased in $\mathrm{PAH}$ patients, contributing to diastolic dysfunction, it could be detrimental for the heart to increase calcium sensitivity even further. Tirasemtiv is a novel calcium sensitiser that is currently in a phase $2 \mathrm{~B}$ clinical trial. It specifically targets fast-twitch muscle fibres and has no effect on cardiac muscle [85]. As smooth muscle cells do not contain troponin, it also has no effect on the pulmonary vasculature. An analogue of Tirasemtiv was tested on single permeabilised muscle fibres of the diaphragm of PAH patients. It greatly improved submaximal force generation of fast-twitch muscle fibres [50]. Besides improving submaximal force generation, calcium sensitisers might also be energetically beneficial, as less calcium is needed for a certain force response. Reuptake of calcium from the cytoplasm into the SR during relaxation is a highly energy-consuming process. It was shown that neuromechanical efficiency improved by $21 \%$ in the diaphragm of healthy controls after acute supplementation of a calcium sensitiser [86].

In summary, ExT including IMT could be beneficial for both cardiac and skeletal muscle function. Nonetheless, improving muscle oxygenation by microRNA-126 modulation and contractile function with calcium sensitisers might further improve exercise capacity and quality of life of PAH patients.

\section{Summary}

Here, we have provided evidence indicating that PAH is not limited to changes in the pulmonary circulation and the RV, but also involves alterations of the LV and skeletal muscle function, which contribute to exercise intolerance. In figure 3, a schematic overview is provided to illustrate how PAH affects these striated muscles. In $\mathrm{PAH}$, the RV adapts to the increase in afterload and becomes hypertrophic, but eventually dilates and fails. This affects LV filling, caused by septum bulging and reduced RV output. The reduction in LV filling leads to LV atrophy and contractile dysfunction. The reduction in both RV and LV function leads to a decrease in cardiac output, which results in an impaired oxygen supply to the skeletal muscles. Furthermore, PAH and RV dysfunction lead to alterations in systemic factors [17]. For example, an increased inflammatory response and neurohormonal overstimulation are present in PAH patients. Together with a reduction in oxygen supply, this affects skeletal muscle function. In addition, we propose that a change in skeletal muscle activity might amplify skeletal muscle dysfunction. Inspiratory muscle activity increases, leading to a substantial reduction in inspiratory muscle strength and diaphragm weakness. However, peripheral muscle activity might decrease which could lead to different adaptations of the peripheral muscles. The reduction in cardiac output, together with peripheral and inspiratory muscle weakness, may contribute to exercise intolerance in PAH patients. Therapeutic strategies targeting striated muscle function may lead to improvements in exercise capacity and quality of life of PAH patients.

\section{References}

1 Rain S, Handoko ML, Westerhof N, et al. Right ventricular diastolic impairment in patients with pulmonary arterial hypertension. Circulation 2013; 128: 2016-2025. 
Rain S, Bos DDSG, Handoko ML, et al. Protein changes contributing to right ventricular cardiomyocyte diastolic dysfunction in pulmonary arterial hypertension. J Am Heart Assoc 2014; 3: e000716.

3 Van de Veerdonk MC, Kind T, Marcus JT, et al. Progressive right ventricular dysfunction in patients with pulmonary arterial hypertension responding to therapy. J Am Coll Cardiol 2011; 58: 2511-2519.

4 Vonk-Noordegraaf A, Haddad F, Chin KM, et al. Right heart adaptation to pulmonary arterial hypertension. J Am Coll Cardiol 2013; 62: D22-D33.

5 Manders E, Bogaard HJ, Handoko ML, et al. Contractile dysfunction of left ventricular cardiomyocytes in patients with pulmonary arterial hypertension. J Am Coll Cardiol 2014; 64: 28-37.

6 Tonelli AR, Plana JC, Heresi GA, et al. Prevalence and prognostic value of left ventricular diastolic dysfunction in idiopathic and heritable pulmonary arterial hypertension. Chest 2012; 141: 1457-1465.

7 Kabitz HJ, Schwoerer A, Bremer HC, et al. Impairment of respiratory muscle function in pulmonary hypertension. Clin Sci 2008; 114: 165-171.

8 De Man FS, van Hees HWH, Handoko ML, et al. Diaphragm muscle fiber weakness in pulmonary hypertension. Am J Respir Crit Care Med 2011; 183: 1411-1418.

9 Mainguy V, Maltais FF, Saey D, et al. Peripheral muscle dysfunction in idiopathic pulmonaryarterial hypertension. Thorax 2010; 65: 113-117.

10 Widmaier EP, Raff H, Strang KT. Vander's Human Physiology: Mechanisms of Body Function. 11th Edn. Columbus, McGraw-Hill, 2008.

11 LeWinter MM, Granzier H. Cardiac titin: a multifunctional giant. Circulation 2010; 121: 2137-2145.

12 Harvey PA, Leinwand LA. The cell biology of disease: cellular mechanisms of cardiomyopathy. J Cell Biol 2011; 194: 355-365.

13 Hoppeler H, Baum O, Lurman G, et al. Molecular mechanisms of muscle plasticity with exercise. Compr Physiol 2011; 1: 1383-1412.

14 Weiner RB, Baggish AL. Exercise-induced cardiac remodeling. Prog Cardiovasc Dis 2012; 54: 380-386.

15 Perhonen MA, Franco F, Lane LD, et al. Cardiac atrophy after bed rest and spaceflight. J Appl Physiol 2001; 91: 645-653.

16 Trappe S, Trappe T, Gallagher P, et al. Human single muscle fibre function with 84 day bed-rest and resistance exercise. J Physiol 2004; 557: 501-513.

17 Rain S, Handoko ML, Vonk Noordegraaf A, et al. Pressure-overload-induced right heart failure. Pflugers Arch 2014; 466: 1055-1063.

18 Haddad F, Doyle R, Murphy DJ, et al. Right ventricular function in cardiovascular disease, part II: pathophysiology, clinical importance, and management of right ventricular failure. Circulation 2008; 117: 1717-1731.

19 Gan CTJ, Lankhaar JW, Marcus JT, et al. Impaired left ventricular filling due to right-to-left ventricular interaction in patients with pulmonary arterial hypertension. Am J Physiol Heart Circ Physiol 2006; 290: H1528-H1533.

20 Hardziyenka M, Campian ME, Reesink HJ, et al. Right ventricular failure following chronic pressure overload is associated with reduction in left ventricular mass evidence for atrophic remodeling. J Am Coll Cardiol 2011; 57: 921-928.

21 Kasner M, Westermann D, Steendijk P, et al. Left ventricular dysfunction induced by nonsevere idiopathic pulmonary arterial hypertension: a pressure-volume relationship study. Am J Respir Crit Care Med 2012; 186: 181-189.

22 Gurudevan SV, Malouf PJ, Auger WR, et al. Abnormal left ventricular diastolic filling in chronic thromboembolic pulmonary hypertension: true diastolic dysfunction or left ventricular underfilling? J Am Coll Cardiol 2007; 49: 1334-1339.

23 Xie GY, Lin CS, Preston HM, et al. Assessment of left ventricular diastolic function after single lung transplantation in patients with severe pulmonary hypertension. Chest 1998; 114: 477-481.

24 Toyooka S, Kusano KF, Goto K, et al. Right but not left ventricular function recovers early after living-donor lobar lung transplantation in patients with pulmonary arterial hypertension. J Thorac Cardiovasc Surg 2009; 138: $222-226$.

25 Kamler M, Herold U, Piotrowski J, et al. Severe left ventricular failure after double lung transplantation: pathophysiology and management. J Heart Lung Transpl 2004; 23: 139-142.

26 Bando K, Keenan RJ, Paradis IL, et al. Impact of pulmonary hypertension on outcome after single-lung transplantation. Ann Thorac Surg 1994; 58: 1336-1342.

27 De Man FS, Tu L, Handoko ML, et al. Dysregulated renin-angiotensin-aldosterone system contributes to pulmonary arterial hypertension. Am J Respir Crit Care Med 2012; 186: 780-789.

28 De Man FS, Handoko ML, Guignabert C, et al. Neurohormonal axis in patients with pulmonary arterial hypertension: friend or foe? Am J Respir Crit Care Med 2013; 187: 14-19.

29 Bristow MR, Minobe W, Rasmussen R, et al. $\beta$-Adrenergic neuroeffector abnormalities in the failing human heart are produced by local rather than systemic mechanisms. J Clin Invest 1992; 89: 803-815.

30 Dorfmuller P, Perros F, Balabanian K, et al. Inflammation in pulmonary arterial hypertension. Eur Respir J 2003; 22: 358-363.

31 Hassoun PM, Mouthon L, Barbera JA. Inflammation, growth factors and pulmonary vascular remodeling. $J$ Am Coll Cardiol 2009; 54: Suppl., S10-S19.

32 Quarck R, Nawrot T, Meyns B, et al. C-reactive protein: a new predictor of adverse outcome in pulmonary arterial hypertension. J Am Coll Cardiol 2009; 53: 1211-1218.

33 Gullestad L, Ueland T, Vinge LE, et al. Inflammatory cytokines in heart failure: mediators and markers. Cardiology 2012; 122: 23-35.

34 Overbeek MJ, Lankhaar JW, Westerhof N, et al. Right ventricular contractility in systemic sclerosis-associated and idiopathic pulmonary arterial hypertension. Eur Respir J 2008; 31: 1160-1166.

35 Overbeek MJ, Mouchaers KTB, Niessen HM, et al. Characteristics of interstitial fibrosis and inflammatory cell infiltration in right ventricles of systemic sclerosis-associated pulmonary arterial hypertension. Int J Rheumatol 2010; 2010: 604615.

36 Lumens J, Blanchard DG, Arts T, et al. Left ventricular underfilling and not septal bulging dominates abnormal left ventricular filling hemodynamics in chronic thromboembolic pulmonary hypertension. Am J Physiol Heart Circ Physiol 2010; 299: H1083-H1091. 
37 Jaijee S, O’Rourke R, Puranik R, et al. Right to left ventricular volume ratio: A novel marker of disease severity in chronic thromboembolic pulmonary hypertension. IJC Heart Vessel 2013; 1: 17-21.

38 Marcus JT, Gan CTJ, Zwanenburg JJM, et al. Interventricular mechanical asynchrony in pulmonary arterial hypertension: left-to-right delay in peak shortening is related to right ventricular overload and left ventricular underfilling. J Am Coll Cardiol 2008; 51: 750-757.

39 McArdle WD, Katch FI, Katch VL. Exercise Physiology: Nutrition, Energy, and Human Performance. Philadelphia, Lippincott Williams \& Wilkins, 2010.

40 Holverda S, Gan CTJ, Marcus JT, et al. Impaired stroke volume response to exercise in pulmonary arterial hypertension. J Am Coll Cardiol 2006; 47: 1732-1733.

41 Provencher $\mathrm{S}$, Chemla D, Hervé $\mathrm{P}$, et al. Heart rate responses during the 6-minute walk test in pulmonary arterial hypertension. Eur Respir J 2006; 27: 114-120.

42 Sun XG, Hansen JE, Oudiz RJ, et al. Exercise pathophysiology in patients with primary pulmonary hypertension. Circulation 2001; 104: 429-435.

43 Trip P, Nossent EJ, de Man FS, et al. Severely reduced diffusion capacity in idiopathic pulmonary arterial hypertension: patient characteristics and treatment responses. Eur Respir J 2013; 42: 1575-1585.

44 Meyer FJ, Lossnitzer D, Kristen AV, et al. Respiratory muscle dysfunction in idiopathic pulmonary arterial hypertension. Eur Respir J 2005; 25: 125-130.

45 Breda AP, Pereira de Albuquerque AL, Jardim C, et al. Skeletal muscle abnormalities in pulmonary arterial hypertension. PLoS One 2014; 9: e114101.

46 Bauer R, Dehnert C, Schoene P, et al. Skeletal muscle dysfunction in patients with idiopathic pulmonary arterial hypertension. Respir Med 2007; 101: 2366-2369.

47 Esposito F, Mathieu-Costello O, Shabetai R, et al. Limited maximal exercise capacity in patients with chronic heart failure: partitioning the contributors. J Am Coll Cardiol 2010; 55: 1945-1954.

48 Ahn B, Empinado HM, Al-Rajhi M, et al. Diaphragm atrophy and contractile dysfunction in a murine model of pulmonary hypertension. PLoS One 2013; 8: 1-7.

49 Manders E, de Man FS, Handoko ML, et al. Diaphragm weakness in pulmonary arterial hypertension: role of sarcomeric dysfunction. Am J Physiol Lung Cell Mol Physiol 2012; 303: L1070-L1078.

50 Manders E, Bonta PI, Kloek JJ, et al. Diaphragm dysfunction in pulmonary arterial hypertension patients. Am J Respir Crit Care Med 2014; 189: A6347.

51 Vescovo G, Ceconi C, Bernocchie P, et al. Skeletal muscle myosin heavy chain expression in rats with monocrotaline induced cardiac hypertrophy and failure. Relation to blood flow and degree of muscle atrophy. Cardiovasc Res 1998; 39: 233-241.

52 Wüst RCI, Myers DS, Stones R, et al. Regional skeletal muscle remodeling and mitochondrial dysfunction in right ventricular heart failure. Am J Physiol Heart Circ Physiol 2012; 302: H402-H411.

53 Potus F, Malenfant S, Graydon C, et al. Impaired angiogenesis and peripheral muscle microcirculation loss contribute to exercise intolerance in pulmonary arterial hypertension. Am J Respir Crit Care Med 2014; 190: 318-328.

54 Batt J, Ahmed SS, Correa J, et al. Skeletal muscle dysfunction in idiopathic pulmonary arterial hypertension. Am J Respir Cell Mol Biol 2014; 50: 74-86.

55 Manders E, Ruiter G, Bogaard H-J, et al. Quadriceps muscle fibre dysfunction in patients with pulmonary arterial hypertension. Eur Respir J 2015; 45: 1737-1740.

56 Gosker HR, Wouters EFM, van der Vusse GJ, et al. Skeletal muscle dysfunction in chronic obstructive pulmonary disease and chronic heart failure: underlying mechanisms and therapy perspectives. Am J Clin Nutr 2000; 71: 1033-1047.

57 Soon E, Holmes AM, Treacy CM, et al. Elevated levels of inflammatory cytokines predict survival in idiopathic and familial pulmonary arterial hypertension. Circulation 2010; 122: 920-927.

58 Reid MB, Lännergren J, Westerblad $\mathrm{H}$. Respiratory and limb muscle weakness induced by tumor necrosis factor- $\alpha$ : involvement of muscle myofilaments. Am J Respir Crit Care Med 2002; 166: 479-484.

59 Li Y, Chen Y, John J, et al. TNF- $\alpha$ acts via p38 MAPK to stimulate expression of the ubiquitin ligase atrogin1/ MAFbx in skeletal muscle. FASEB J 2005; 19: 362-370.

60 Batt J, Ahmed SS, Correa J, et al. Skeletal muscle dysfunction in idiopathic pulmonary arterial hypertension. Am J Respir Cell Mol Biol 2014; 50: 74-86.

61 Yoshida T, Galvez S, Tiwari S, et al. Angiotensin II inhibits satellite cell proliferation and prevents skeletal muscle regeneration. J Biol Chem 2013; 288: 23823-23832.

62 Gorman JL, Liu STK, Slopack D, et al. Angiotensin II evokes angiogenic signals within skeletal muscle through co-ordinated effects on skeletal myocytes and endothelial cells. PLoS One 2014; 9: e85537.

63 D'Antona G, Pellegrino MA, Adami R, et al. The effect of ageing and immobilization on structure and function of human skeletal muscle fibres. J Physiol 2003; 552: 499-511.

64 Naeije R. Breathing more with weaker respiratory muscles in pulmonary arterial hypertension. Eur Respir J 2005; 25: 6-8.

65 Mantilla CB, Sieck GC. Plasticity in respiratory motor control. Invited Review:Mechanisms underlying motor unit plasticity in the respiratory system. J Appl Physiol 2003; 94: 1230-1241.

66 Zafrir B. Exercise training and rehabilitation in pulmonary arterial hypertension: rationale and current data evaluation. J Cardiopulm Rehabil Prev 2013; 33: 263-273.

67 Grünig E, Ehlken N, Ghofrani A, et al. Effect of exercise and respiratory training on clinical progression and survival in patients with severe chronic pulmonary hypertension. Respiration 2011; 81: 394-401.

68 Grünig E, Lichtblau M, Ehlken N, et al. Safety and efficacy of exercise training in various forms of pulmonary hypertension. Eur Respir J 2012; 40: 84-92.

69 Kabitz HJ, Bremer HC, Schwoerer A, et al. The combination of exercise and respiratory training improves respiratory muscle function in pulmonary hypertension. Lung 2013; 192: 321-328.

70 Mereles D, Elkhen N, Kreuscher S, et al. Exercise and respiratory training improve exercise capacity and quality of life in patients with severe chronic pulmonary hypertension. Circulation 2006; 114: 1482-1489.

71 Nagel C, Prange F, Guth S, et al. Exercise training improves exercise capacity and quality of life in patients with inoperable or residual chronic thromboembolic pulmonary hypertension. PLoS One 2012; 7: e41603. 
De Man FS, Handoko ML, Groepenhoff H, et al. Effects of exercise training in patients with idiopathic pulmonary arterial hypertension. Eur Respir J 2009; 34: 669-675.

73 Mainguy V, Maltais F, Saey D, et al. Effects of a rehabilitation program on skeletal muscle function in idiopathic pulmonary arterial hypertension. J Cardiopulm Rehabil Prev 2010; 30: 319-323.

74 Dall'Ago P, Chiappa GRS, Guths H, et al. Inspiratory muscle training in patients with heart failure and inspiratory muscle weakness: a randomized trial. J Am Coll Cardiol 2006; 47: 757-763.

75 Marco E, Ramírez-Sarmiento AL, Coloma A, et al. High-intensity vs. sham inspiratory muscle training in patients with chronic heart failure: a prospective randomized trial. Eur J Heart Fail 2013; 15: 892-901.

76 Mello PR, Guerra GM, Borile S, et al. Inspiratory muscle training reduces sympathetic nervous activity and improves inspiratory muscle weakness and quality of life in patients with chronic heart failure: a clinical trial. J Cardiopulm Rehabil Prev 2012; 32: 255-261.

77 Handoko ML, de Man FS, Happe CM, et al. Opposite effects of training in rats with stable and progressive pulmonary hypertension. Circulation 2009; 120: 42-49.

78 Caron AZ, Haroun S, Leblanc E, et al. The proteasome inhibitor MG132 reduces immobilization-induced skeletal muscle atrophy in mice. BMC Musculoskelet Disord 2011; 12: 185

79 van Hees HWH, Li YP, Ottenheijm CAC, et al. Proteasome inhibition improves diaphragm function in congestive heart failure rats. Am J Physiol Lung Cell Mol Physiol 2008; 294: L1260-L1268.

80 Agten A, Maes K, Thomas D, et al. Bortezomib partially protects the rat diaphragm from ventilator-induced diaphragm dysfunction. Crit Care Med 2012; 40: 2449-2455.

81 Ruiter G, Ying Wong Y, de Man FS, et al. Right ventricular oxygen supply parameters are decreased in human and experimental pulmonary hypertension. J Heart Lung Transpl 2013; 32: 231-240.

82 Matuszczak Y, Farid M, Jones J, et al. Effects of $\mathrm{N}$-acetylcysteine on glutathione oxidation and fatigue during handgrip exercise. Muscle Nerve 2005; 32: 633-638.

83 Edes I, Kiss E, Kitada Y, et al. Effects of levosimendan, a cardiotonic agent targeted to troponin C, on cardiac function and on phosphorylation and $\mathrm{Ca}^{2+}$ sensitivity of cardiac myofibrils and sarcoplasmic reticulum in guinea pig heart. Circ Res 1995; 77: 107-113.

84 van Hees HWH, Dekhuijzen PNR, Heunks LMA. Levosimendan enhances force generation of diaphragm muscle from patients with chronic obstructive pulmonary disease. Am J Respir Crit Care Med 2009; 179: 41-47.

85 Russell AJ, Hartman JJ, Hinken AC, et al. Activation of fast skeletal muscle troponin as a potential therapeutic approach for treating neuromuscular diseases. Nat Med 2012; 18: 452-455.

86 Doorduin J, Sinderby CA, Beck J, et al. The calcium sensitizer levosimendan improves human diaphragm function. Am J Respir Crit Care Med 2012; 185: 90-95. 